\title{
A dynamic map for learning, communicating, navigating and improving therapeutic development
}

\author{
John Wagner, Andrew M. Dahlem, Lynn D. Hudson, Sharon F. Terry, \\ Russ B. Altman, C. Taylor Gilliland, Christopher DeFeo and Christopher P. Austin
}

Drug discovery and development is a highly challenging process (Nat. Rev. Drug Discov. 9, $203-214 ; 2010)^{1}$. Its common characterization as a 'pipeline' connotes consistent unidirectional flow of a candidate therapeutic in unchanged form from basic research discovery to drug to utilization, in stark contrast to the diverse web of failure-prone iterative learning loops that, in reality, must be traversed by scientists, physicians, regulators, payers and patients to develop and use new treatments ${ }^{2}$. This deeply engrained misnomer contributes to the lack of public understanding of the drug development process, with implications for science and public policy, and leads to actions that may fail to improve, and perhaps even worsen, the process.

The Forum on Drug Discovery, Development, and Translation of the US National Academies of Sciences, Engineering, and Medicine is a platform for dialogue and collaboration among thought leaders in government, academia, industry, foundations, and disease and patient advocacy focused on improving the system of therapeutic discovery. Here, we report the output of a yearlong effort by an 'Action Collaborative' of this forum, augmented by a diverse group of additional experts (see Acknowledgements), to conceptualize, create and annotate an accurate and customizable 'Drug Discovery, Development and Deployment Map' (4DM) of the process (FIG. 1).

\section{Beyond the chevron}

The 'chevron' conceptualization of drug development is a common, schematic representation of the pipeline concept. Previous efforts to more accurately portray the drug development process, including the excellent 'NETS' map of Baxter et al. ${ }^{2}$, took major steps to zoom in on each step of the chevron and display a network of interacting activity domains. In the $4 \mathrm{DM}$, this concept is extended, converting the components of the traditional chevron diagram into 'neighbourhoods' of activities. For example, the clinical research and development neighbourhood of the 4DM (FIG. 1e) corresponds to the clinical development segment of the chevron. However, each neighbourhood highlights the myriad of routes and steps involved, as well as how these activities interact with those in other neighbourhoods - for example, deposition of biospecimens from clinical studies (FIG. 1e) into biorepositories to be utilized for target identification (FIG. 1a) - which are not discernible in the stepwise linear representation of the chevron diagram.

Aspects of therapeutic development shown in the $4 \mathrm{DM}$ that are missing entirely from the chevron include: a neighbourhood for biomarker development (FIG. 1 b), which takes place over multiple stages of the development process; inclusion of natural history studies, epidemiology and patient input in the clinical research and development neighbourhood (FIG. 1e); a post-marketing neighbourhood (FIG. $1 \mathrm{~g}$ ) that includes observations on safety, usage patterns and effectiveness; and a medical landscape neighbourhood (FIG. $1 \mathrm{~h}$ ) covering the increasingly important issues of access and reimbursement.

Within neighbourhoods, a distinction is made between steps that can be performed in parallel and those that must be performed in series. The failure to progress, which is so common in therapeutic development, is reflected in reiterative loops to indicate that many 'shots on goal' are often required before success is achieved at any step. We also attempted to indicate that discoveries at one step of the process may render earlier results irrelevant or unreliable, or even create new routes that provide shortcuts to later stages. Finally, since the development processes for small molecules and biologics differ in substantial ways, distinct versions of the 4DM were created, using monoclonal antibodies as the representative biologic ${ }^{3}$. The major differences between these versions are in neighbourhoods $\mathrm{c}$ and $\mathrm{d}$, which cover therapeutic candidate identification and optimization, respectively.

\section{Evolution and application of the 4DM}

For centuries, maps were static, paper-based and non-interactive. The last decade has seen the transformation of maps into dynamic, multi-parameter, customizable electronic tools with features that include varying magnification and indications of limitations to efficient navigation that other travellers have reported. We have begun to incorporate these features into the 4DM and others will be refined in future versions. The current version of the $4 \mathrm{DM}$ can be viewed with or without 'traffic' indicated. To develop the latter, the authors, other members of the forum and a group of experts invited to participate (see Acknowledgements) voted on what steps they and their organizations had found severely or moderately onerous (red, pink or orange outlined boxes in FIG. 1, respectively; see also Supplementary information S1 (figure)) in terms of the time required, resources or failure rate.

The steps outlined in red - selection of therapeutic targets, biomarker qualification, recruitment of clinical trial participants and incorporation of the patient perspective into the therapeutic development process - will be familiar to those well versed in the drug development process as being refractory to generally successful approaches, despite enormous investment of time, effort, and resources. Some of the steps indicated as being problematic but less so (outlined in orange) include commonly appreciated roadblocks (for example, identification of biomarkers), but others may be less familiar (for example, the time required for contractual and legal agreements, which is growing in importance given the increasingly multi-party nature of drug development projects).

The 4DM will be of value to a wide variety of stakeholders. First, for scientists, patient advocates, policymakers or members of the public who are new to drug development, the $4 \mathrm{DM}$ will provide orientation to the realities of the process. For educators, it will provide a freely available teaching tool. For those embarking on a drug development project, it will help to locate the state of the project, identify next steps and anticipate downstream challenges. For those working to improve the process via translational science projects, the $4 \mathrm{DM}$ will indicate bottlenecks in need of innovation to expedite the process of new medicine discovery.

Already, use of the map by stakeholders convened under the auspices of the forum has focused attention on how bottlenecks and blockages both hinder therapeutic development as a whole and how they are unique to different sectors. This type of connection can drive understanding and promote dialogue in more thoughtful and unanticipated areas. However, the map itself will need to be updated by all stakeholders involved in 


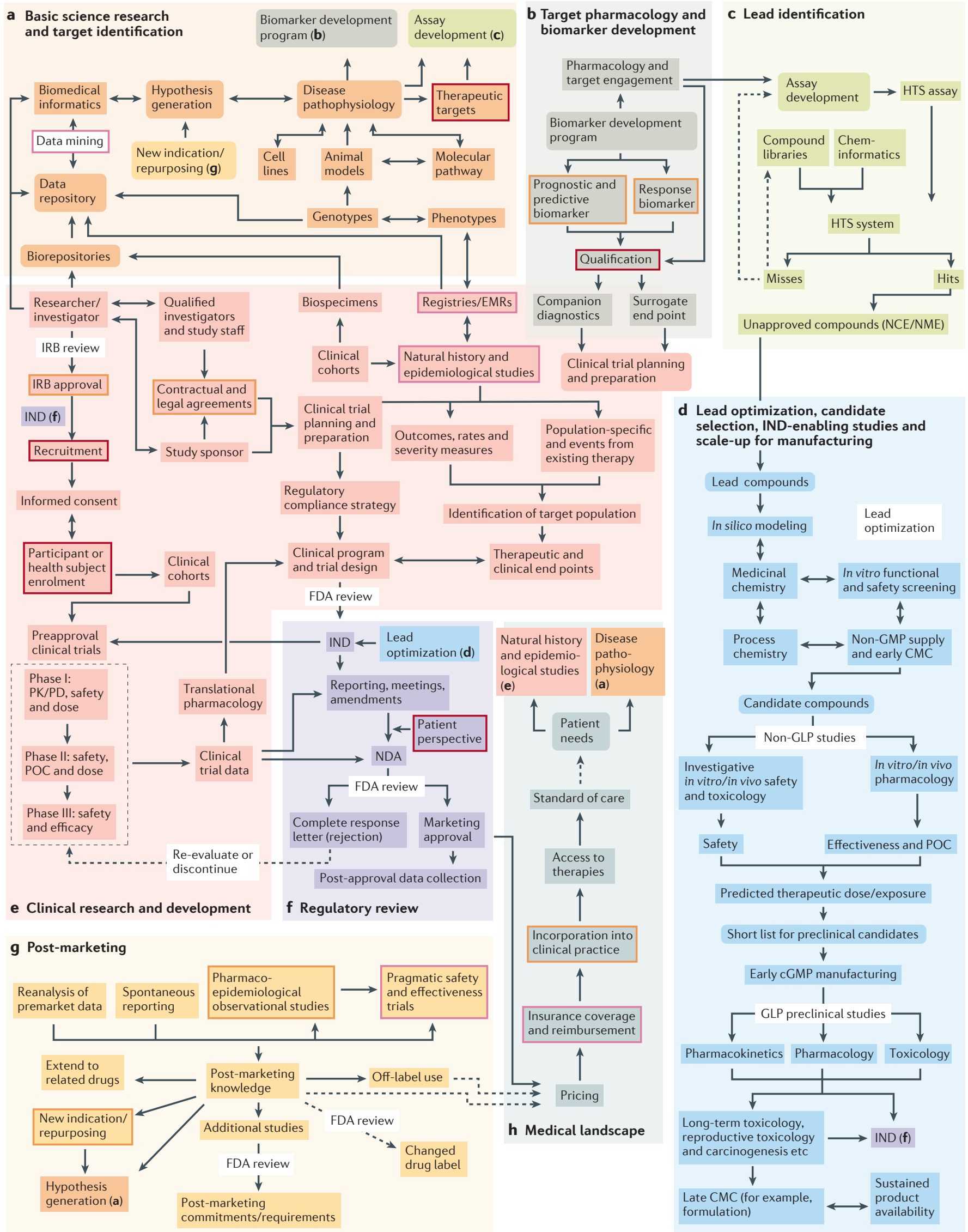


« Figure 1 | 4DM map for small-molecule drug development. The map comprises neighbourhoods (panels $\mathbf{a}-\mathbf{h}$ ), each of which consists of a complex network of steps that interact with steps in other neighbourhoods. Steps identified in the mapping process as being characterized by the greatest cost, time or likelihood of failure are identified with a red outline; less problematic roadblocks are identified with a pink or orange outline (see supplementary information S1 (figure) for a version with the identified steps only highlighted). Dotted lines indicate possible alternative pathways between two steps. A version of this map is available to download from the National Center for Advancing Translational Sciences website and is licensed to the public under the Creative Commons Attribution-Share Alike 4.0 license, which allows use and adaptation as long as the user provides attribution and shares any adaptations back to the public under the same license. CMC, chemistry, manufacturing and controls; EMR, electronic medical record; FDA, Food and Drug Administration; GLP, good laboratory practice; GMP, good manufacturing practice; HTS, high-throughput screening; IND, investigational new drug; IRB, institutional review board; NCE, new chemical entity; NDA, new drug application; NME, new molecular entity; PK/PD, pharmacokinetics/pharmacodynamics; POC, proof of concept.

therapeutic development so that a shared vocabulary evolves that will facilitate problem solving and promote innovation.

The process of creating the $4 \mathrm{DM}$ raised as many questions as it answered. This reflects the diversity of viewpoints and largely anecdotal nature underlying our understanding of the fundamental roadblocks to improved therapeutic development. It also became clear that there is a wide range of preference with regard to the simplicity-complexity continuum, with some users preferring a simpler and more streamlined map and others a more dynamic and complex one. Thus, we present the $4 \mathrm{DM}$ as the start, not the completion, of what should be an increasingly broad, crowdsourced effort to understand and improve the therapeutic development process.

The map enables a dialogue among stakeholders for discussion of where bottlenecks exist for innovation and offers the potential for collaborative action to expedite the overall process of new medicine discovery. Clearly, the map could be refined by making it more dynamic, allowing more or less detail depending on the question of interest and by incorporating other critical variables such as time and cost. We fully expect that the map will be refined to include other perspectives and new features. A version of the map is freely available via a Creative Commons Attribution-Share Alike 4.0 license from the National Center for Advancing Translational Sciences website, in order to allow anyone to use, display, distribute, customize or adapt it for their use.

We live in an era of enormous promise for therapeutic development and unprecedented demand for new therapies, combined with the entry of many new participants in the process. While this is cause for celebration, a common understanding and language of the topography of therapeutic development and its difficulties is essential to prevent unrealistic expectations and focus our collective energy on system-wide improvements that will benefit all. Toward this goal, we welcome input on how to make the map more accurate, comprehensive and useful to all stakeholders.

John Wagner is at Takeda Pharmaceuticals, Cambridge, Massachusetts 02142, USA

Andrew M. Dahlem is at Lilly Research Laboratories, Eli Lilly and Company, Indianapolis, Indiana 46285, USA.
Lynn D. Hudson is at the Critical Path Institute, Tucson, Arizona 85718, USA.

Sharon F. Terry is at the Genetic Alliance, Washington, DC 20008, USA.

Russ B. Altman is at Stanford University, Stanford, California 94305, USA.

C. Taylor Gilliland and Christopher P. Austin are at the National Center for Advancing Translational Sciences, National Institutes of Health, Bethesda, Maryland 20817, USA

Christopher DeFeo is at Georgetown Preparatory School, North Bethesda, Maryland 20852, USA.

Correspondence to C.P.A. austinc@mail.nih.gov doi:10.1038/nrd.2017.217 Published online 22 Dec 2017

1. Paul, S. M. et al. How to improve R\&D productivity: the pharmaceutical industry's grand challenge. Nat. Rev. Drug Discov. 9, 203-214 (2010).

2. Baxter, K. et al. An end to the myth: there is no drug development pipeline. Sci. Transl Med. 5, $171 \mathrm{~cm} 1$ (2013).

3. Wagner, J. A. et al. Application of a dynamic map for learning, communicating, navigating and improving therapeutic development. Clin. Trans/ Sci. (in the press: doi: $10.1111 /$ cts. 12531)

\section{Acknowledgements}

The authors are grateful to the diverse group of experts who provided valuable contributions through numerous teleconferences and in-person meetings, including C. Adams, M. Anderson, J. Berlin, J. Bernd, P. Burgoon, R. Califf, T. Coyle, M. Culp, J. Drazen, S. Galson, J. Gillon, K. Gottesdiener, H. Greenberg, A. Heatherington, S. Heidel, S.C. Johnston, P. Kaufmann, J. Kehne, R. Kelley, R. Krall, K. Lambertson, F. Lewis-Hall, M. Lim, S. McCune, S. McGoohan, R. Moscicki, B. Munos, R. Murray, J. Orloff, T. Oprea, D. Reese, I. Robinson, S. Ryder, J. Scott, L. Skirboll, B. Smith, L. Stoner, B. Strom, P.K. Tandon, D. Thomas, D. Throckmorton, S. Weir, L. Winsky, C. Wolinetz, J. Woodcock, V. Wroblewski and B. Yao. The authors also thank the Forum on Drug Discovery, Development, and Translation staff, including M. Boname, E. Busta, A. Wagner Gee and A. Claiborne, for their assistance.

Competing interests statement

The authors declare no competing interests.

FURTHER INFORMATION

Drug Discovery, Development and Deployment Map (4DM):

https://ncats.nih.gov/translation/maps

SUPPLEMENTARY INFORMATION

See online article: $\underline{\mathrm{S} 1}$ (figure)

ALL LINKS ARE ACTIVE IN THE ONLINE PDF 